\title{
Spatial Diversity Performance of Multiport Antennas in the Presence of a Butler Network
}

\author{
Nima Jamaly, Member, IEEE, Anders Derneryd, Senior Member, IEEE and Yahya Rahmat-Samii, Fellow, IEEE
}

\begin{abstract}
The main concern of this paper is to study the presence of a cascaded Butler network in diversity antenna systems. The advantages of a cascaded Butler network in 2D multipath environments have been already shown. These advantages are limited to either correlated or nonuniform multipath environments with a line-of-sight component. In the frame of this paper, for the first time, we show a further advantage of a cascaded Butler network in 3D multipath environments, including the effects of coupling. This advantage is associated with an improved convergence in received signals' covariance matrix in a multipath environment wherein the number of independent incoming waves is limited, i.e., non-rich multipath environment. For this purpose, we use a simulation tool in which a multipath scenario with certain number of incoming waves is emulated and some selected multiport antennas are exposed to it. Afterwards, the simulated random received signals are used to yield different performance metrics like spatial correlation. This advantage achieves considerable practical interest since a typical actual multipath scenario is most likely a non-rich multipath environment.
\end{abstract}

Index Terms-Multiport antennas, Butler network, beamforming, multiport matching efficiency, effective diversity gain, richness threshold.

\section{INTRODUCTION}

D ISCRETE Fourier transformation is a contemporary concern for its advantages in multiple-input and multipleoutput (MIMO) wireless communication systems. In practice, a discrete Fourier transformation over spatially received signals can be realized in the RF domain by an ideal Butler network [1]. Firstly, in [1] the authors studied the effects of implementing a Butler network in a MIMO communication system in both correlated and uncorrelated multipath environments. From a communication engineer's point of view, they showed the benefits of a cascaded Butler network in correlated multipath environments. Later, A. Grau et al. investigated the matter from an antenna standpoint and highlighted the advantages inherent in using a Butler network [2]-[4].

However, some additional developments of the aforementioned works may become useful. For instance, the multipath model used to simulate their results is in $2 \mathrm{D}$, e.g. a two-ring model located at the transmitter and receiver with the scatterers distributed uniformly in the azimuth plane [2], [3]. Moreover, the developments appear to be limited to cases of negligible coupling among radiating elements. In contrast, we simulate

N. Jamaly is with the Department of Signals and Systems, Chalmers University of Technology, Gothenburg, SE-412 96 Sweden; e-mail: jamaly@chalmers.se

A. Derneryd is with the Ericsson AB, Gothenburg, Sweden. He is also an adjunct professor at Lund University, Sweden.

Y. Rahmat-Samii is with the University of California, Los Angeles, USA. some proposed antennas in 3D multipath environments including coupling among nearby radiating elements. In addition to these, we analyze the behavior of the proposed multiport antennas in the presence and absence of a Butler network in two distinct multipath environments: $(a)$ a rich multipath environment wherein the received signals at different ports of the antennas have converged joint probability density function and $(b)$ a multipath environment with finite number of incident waves which is, henceforth, referred to as a non-rich multipath environment [5].

In general, the advantages of a cascaded Butler network can be viewed from two perspectives. From the first standpoint, an advantage is linked to an improved mean effective gain (MEG). This advantage is likely only realizable in certain non-uniform multipath environments with limited angular spread, regardless of the number of incident waves [4]. On the contrary, in this paper, we reveal a further advantage of a Butler network in diversity antenna systems, which to the best of our knowledge has not been appreciated before. In brief, it is clarified that in general the spatial correlation between the received random signals depends also on the number of incident waves. We reveal that a Butler network facilitates the convergence of the spatial correlation and thus can potentially enhance the overall diversity performance in a non-rich multipath environment.

Throughout this paper we restrict ourselves to uniform multipath environments in order to focus on the effects of a cascaded Butler network solely upon correlation convergence and not MEG enhancement. Even so, the extracted conclusions are general and hold for non-uniform multipath environments too. This study ultimately leads to a fundamental conclusion saying that, as long as the diversity gain is concerned, the shape of the embedded patterns plays more critical role than the element separation in non-rich multipath environments. We stress that our study is limited to random received signals which have converged Rayleigh distributions.

The structure of the paper is as follows. Section II develops a general compact formula to calculate the total embedded efficiencies in the presence of an arbitrary microwave network. The total embedded efficiencies are used to normalize the associated embedded patterns, which play an important role in later sections. A general description of the microwave component under study as well as the simulation approach is presented in Section III. Section IV addresses the effects of Butler network on diversity gain performance in a rich multipath environment. This is followed in Section V applying a similar study in a non-rich multipath circumstance.

To set the notation, letters with an over-bar sign denote the column vectors, whereas the bold letters represent matrices. 
The matrix I denotes the identity matrix. The dagger sign .$^{\dagger}$ stands for Hermitian transpose while the superscript $T$ for transpose. The incident and reflected wave vectors are normalized such that their square is the associated mean power [6, pp. 182].

\section{Multiport Matching EfFiciency Formulation}

In order to normalize the far field patterns used in our simulations we need to know the total embedded element efficiencies. In lossless structures, the total embedded element efficiencies equate the multiport matching efficiencies [7]. Thus, the main goal in this section is to derive a compact formula for multiport matching efficiency in an $n$-port antenna system in the presence of an arbitrary passive microwave network. Indeed, the details presented here are the base for generalization of multiport matching efficiency in the presence of two or more number of cascaded networks [8]. We start with briefly reviewing the antenna modeling upon which the required formulations are developed.

\section{A. Antenna Modeling: Background}

Assume an $n$-port antenna system characterized by its scattering matrix, $\mathbf{S}_{\text {ant }}$. The antenna's scattering matrix can be divided into four submatrices, as shown in (1) [9], [10]. Submatrix $\mathbf{S}_{a}$ contains the input reflection coefficients as well as the coupling among different element ports. Regarding the other submatrices, which are all functions of angular direction, $\mathbf{S}_{b}$ and $\mathbf{S}_{c}$ link the element ports with the two orthogonal radiation ports, whereas submatrix $\mathbf{S}_{d}$ describes the structural antenna scattering with the element ports being matchedterminated.

$$
\mathbf{S}_{\text {ant }}=\left[\begin{array}{ll}
\mathbf{S}_{a(n \times n)} & \mathbf{S}_{b(n \times 2)} \\
\mathbf{S}_{c(2 \times n)} & \mathbf{S}_{d(2 \times 2)}
\end{array}\right]_{(n+2) \times(n+2)}
$$

To construct a general formula for the multiport matching efficiency, we are solely concerned about the input ports' scattering matrix, $\mathbf{S}_{a}$. In a similar way, the middle microwave network in Fig. 1 is characterized by its S-matrix, $\mathbf{S}_{m}$. The sub-blocks of this matrix are identified as

$$
\mathbf{S}_{m}=\left[\begin{array}{ll}
\mathbf{S}_{11(n \times n)} & \mathbf{S}_{12(n \times n)} \\
\mathbf{S}_{21(n \times n)} & \mathbf{S}_{22(n \times n)}
\end{array}\right]_{(2 n \times 2 n)}
$$

\section{B. Derivation of Multiport Matching Efficiencies}

Multiport matching efficiency is the ratio between the accepted power by a multiport antenna, $P_{\text {acc }}$, and the maximum available power from the source exciting it, $P_{\text {avs }}$ [7]. The primary target is to derive both of them in terms of the source voltages. The necessary parameters in the following derivations are shown in Fig. 1. First, we need to derive an expression for the accepted power. To this end, let us start by expressing the incident waves at the input of the microwave network, $\bar{a}_{m}$, in terms of the sources' normalized voltage vector, $\bar{a}_{s}$. Using the voltage division rule in the fundamental circuit theorem, we can write

$$
\bar{a}_{m}=\left(\mathbf{I}+\boldsymbol{\Gamma}_{1}\right)^{-1} \mathbf{Q}_{m}{ }^{-1} \bar{a}_{s}=\mathbf{T}_{s} \bar{a}_{s}
$$

wherein $\mathbf{Q}_{m}$ is defined as

$$
\mathbf{Q}_{m}=\left[\left(\mathbf{I}+\boldsymbol{\Gamma}_{s}\right)\left(\mathbf{I}-\boldsymbol{\Gamma}_{s}\right)^{-1}\left(\mathbf{I}-\boldsymbol{\Gamma}_{1}\right)\left(\mathbf{I}+\boldsymbol{\Gamma}_{1}\right)^{-1}+\mathbf{I}\right]
$$

with

$$
\boldsymbol{\Gamma}_{s}=\left(\mathbf{Z}_{s}+\mathbf{Z}_{\circ}\right)^{-1}\left(\mathbf{Z}_{s}-\mathbf{Z}_{\circ}\right)
$$

in which $\mathbf{Z}_{\circ}$ is an $n \times n$ diagonal matrix of the characteristic impedance of the system. The matrix $\mathbf{T}_{s}$ can best be called the source transmission matrix. Moreover, the reflection coefficient matrices at both ends of the microwave network are obtained by

$$
\begin{aligned}
& \boldsymbol{\Gamma}_{1}=\mathbf{S}_{11}+\mathbf{S}_{12} \mathbf{S}_{a}\left(\mathbf{I}-\mathbf{S}_{22} \mathbf{S}_{a}\right)^{-1} \mathbf{S}_{21} \\
& \boldsymbol{\Gamma}_{2}=\mathbf{S}_{22}+\mathbf{S}_{21} \boldsymbol{\Gamma}_{s}\left(\mathbf{I}-\mathbf{S}_{11} \boldsymbol{\Gamma}_{s}\right)^{-1} \mathbf{S}_{12}
\end{aligned}
$$

Now, by virtue of (3), the input power to the microwave network, denoted by $P_{m}$, is achieved by

$$
\begin{aligned}
P_{m} & =\bar{a}_{m}^{\dagger} \bar{a}_{m}-\bar{b}_{m}^{\dagger} \bar{b}_{m}=\bar{a}_{m}^{\dagger}\left(\mathbf{I}-\boldsymbol{\Gamma}_{1}^{\dagger} \boldsymbol{\Gamma}_{1}\right) \bar{a}_{m} \\
& =\bar{a}_{s}^{\dagger} \mathbf{T}_{s}^{\dagger}\left(\mathbf{I}-\boldsymbol{\Gamma}_{1}^{\dagger} \boldsymbol{\Gamma}_{1}\right) \mathbf{T}_{s} \bar{a}_{s}
\end{aligned}
$$

On the other hand, the incident waves at the antenna ports are shown to be

$$
\bar{a}_{a}=\left(\mathbf{I}-\mathbf{S}_{22} \mathbf{S}_{a}\right)^{-1} \mathbf{S}_{21} \bar{a}_{m}
$$

Therefore, the accepted power can be written as

$$
\begin{aligned}
P_{\mathrm{acc}} & =\bar{a}_{a}^{\dagger}\left(\mathbf{I}-\mathbf{S}_{a}^{\dagger} \mathbf{S}_{a}\right) \bar{a}_{a} \\
& =\bar{a}_{s}^{\dagger} \mathbf{T}_{t}^{\dagger}\left(\mathbf{I}-\mathbf{S}_{a}^{\dagger} \mathbf{S}_{a}\right) \mathbf{T}_{t} \bar{a}_{s}
\end{aligned}
$$

wherein, by use of (8) and (3), the total transmission coefficient of the cascaded system, $\mathbf{T}_{t}$, is defined as

$$
\mathbf{T}_{t}=\left(\mathbf{I}-\mathbf{S}_{22} \mathbf{S}_{a}\right)^{-1} \mathbf{S}_{21}\left(\mathbf{I}+\boldsymbol{\Gamma}_{1}\right)^{-1} \mathbf{Q}_{m}{ }^{-1}
$$

To achieve the multiport matching efficiency, we also need to obtain the maximum available power from the source. For this purpose, we recall that the accepted power in (7) equals the maximum available power as long as $\boldsymbol{\Gamma}_{1}=\boldsymbol{\Gamma}_{s}{ }^{\dagger}$. That is

$$
\begin{aligned}
P_{\mathrm{avs}} & =\left.P_{m}\right|_{\boldsymbol{\Gamma}_{1}=\boldsymbol{\Gamma}_{s}{ }^{\dagger}} \\
& =\bar{a}_{s}^{\dagger} \mathbf{T}_{m}^{\dagger}\left(\mathbf{I}-\boldsymbol{\Gamma}_{s} \boldsymbol{\Gamma}_{s}^{\dagger}\right) \mathbf{T}_{m} \bar{a}_{s}
\end{aligned}
$$

where

$$
\mathbf{T}_{m}=\left[\left(\mathbf{I}+\boldsymbol{\Gamma}_{s}\right)\left(\mathbf{I}-\boldsymbol{\Gamma}_{s}\right)^{-1}\left(\mathbf{I}-\boldsymbol{\Gamma}_{s}^{\dagger}\right)+\left(\mathbf{I}+\boldsymbol{\Gamma}_{s}^{\dagger}\right)\right]^{-1}
$$

Having obtained all the required parameters, now we can write an expression for the multiport matching efficiency. For a certain excitation scheme, $\bar{a}_{s}$, the multiport matching efficiency becomes

$$
\left.e_{\mathrm{mp}}\right|_{\bar{a}_{s}}=\frac{\bar{a}_{s}^{\dagger} \mathbf{T}_{t}^{\dagger}\left(\mathbf{I}-\mathbf{S}_{a}^{\dagger} \mathbf{S}_{a}\right) \mathbf{T}_{t} \bar{a}_{s}}{\bar{a}_{s}^{\dagger} \mathbf{T}_{m}^{\dagger}\left(\mathbf{I}-\boldsymbol{\Gamma}_{s} \boldsymbol{\Gamma}_{s}^{\dagger}\right) \mathbf{T}_{m} \bar{a}_{s}}
$$




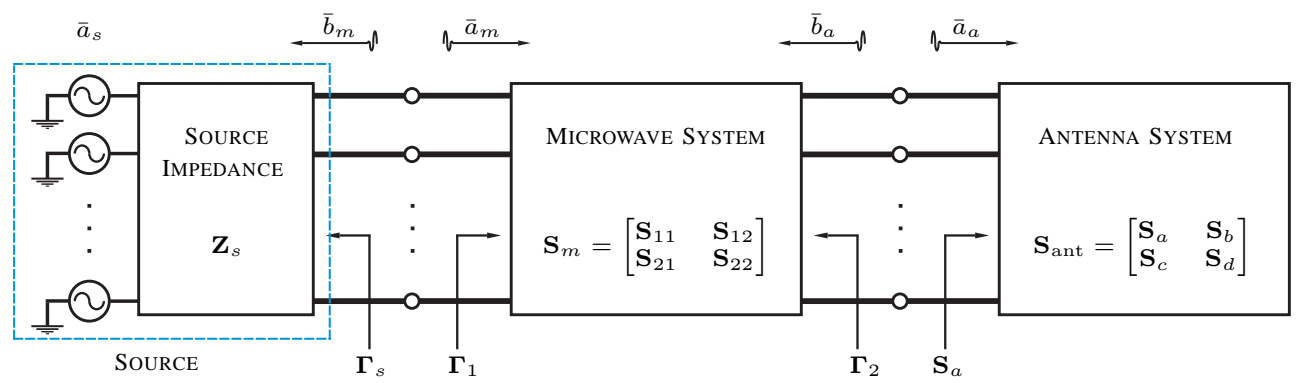

Fig. 1. Microwave circuit model of a multiport antenna system connected to a set of sources through an arbitrary microwave network.

with $\mathbf{T}_{t}$ and $\mathbf{T}_{m}$ defined in (10) and (12), respectively. The definition given above is quite general and can be applied to any arbitrary excitation scheme, $\bar{a}_{s}$. Since the diversity characterization of multiport antennas in receive mode is our main concern, the excitation vector of a single non-zero entry is of particular interest.

As an example, assume we wish to design a matching network for maximizing the multiport matching efficiency, $e_{\mathrm{mp}}$. For this purpose, the ultimate goal is to simultaneously achieve $\boldsymbol{\Gamma}_{1}=\boldsymbol{\Gamma}_{s}^{\dagger}$ and $\boldsymbol{\Gamma}_{2}=\mathbf{S}_{a}^{\dagger}$. Note that, under these constraints, the $\mathbf{S}_{11}$ and $\mathbf{S}_{22}$ in (6) have to be determined such that the associated expressions hold.

\section{Simulation Description}

The goal in this section is to describe and motivate different presumptions in the upcoming simulations upon which we carry out our study. In the first part, the configurations of two multiport antennas, which are chosen arbitrarily, are depicted. In the second subsection, the characteristics of an ideal Butler network are highlighted. Later, we briefly explain the way we obtain different desired parameters from the derived formulas in Section II. A review of the approach used to calculate the received random signals and the associated metrics finalizes this section.

\section{A. Multiport Antennas under Study}

In this study, we arbitrarily choose two different four-port antennas. Firstly, a case of four parallel equidistant monopoles (4MP) above a perfect electric conductor (PEC) plane is investigated. The schematic of this structure is depicted in Fig. 2 in which an arbitrary element separation, $d$, is chosen. The resonance frequency of these identical elements in isolation state is slightly more than $f_{\circ} \approx 1 \mathrm{GHz}\left(\lambda_{\circ} \approx 0.3 \mathrm{~m}\right)$.

The second structure under study is four equidistant horizontal parallel dipoles (4HD) at $h=0.15 \lambda_{\circ}$ height above a PEC plane. The resonance frequency of these similar dipoles while isolated is around that of the preceding counterpart structure. The 4HD radiating elements present higher directivity (e.g., around $7-9 \mathrm{dBi}$ dependent on the element separation and that whether the elements are internal or lateral) in comparison with 4MP.

All simulations are performed using a method of moment full-wave simulator. Note that, although in these figures the PEC plane is shown to be finite, it extents to infinity in our simulations.

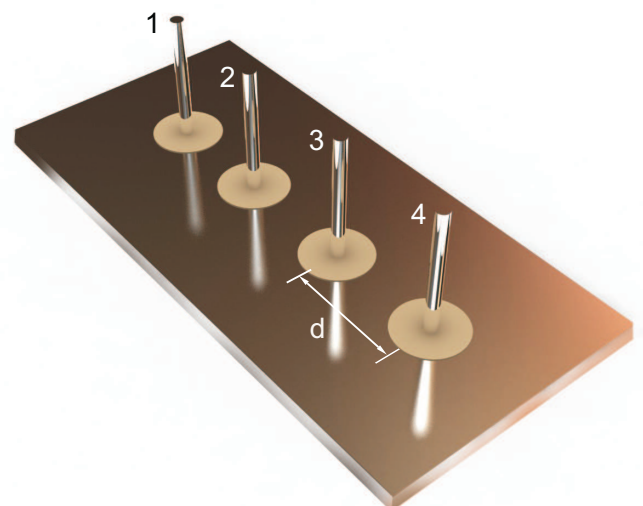

Fig. 2. Four equidistant $\lambda_{\circ} / 4$ monopoles (4MP) above a PEC plane.

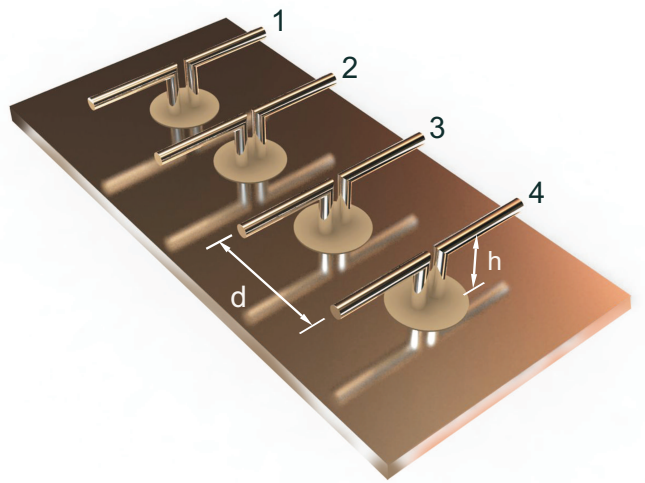

Fig. 3. Four equidistant $\lambda_{\circ} / 2$ dipoles (4HD) above a PEC plane.

\section{B. Ideal Butler Network}

To focus solely on the effects of beam-forming upon the diversity gain performance, we presume an ideal Butler network throughout this paper. The Butler network is a lossless, reciprocal and transparent network (i.e., it is matched at both ends) within the desired bandwidth. Being matched at both ends means that the submatrices $\mathbf{S}_{11}$ and $\mathbf{S}_{22}$ in $\mathbf{S}_{m}$ (Equation (2)) are both zero matrices. The S-matrix of this network is [11]

$$
\mathbf{S}_{m}=\left[\begin{array}{cc}
\mathbf{0} & \mathbf{T} \\
\mathbf{T}^{\top} & \mathbf{0}
\end{array}\right]_{8 \times 8}
$$


wherein

$$
\mathbf{T}=\frac{1}{2}\left[\begin{array}{cccc}
\exp \left(j \frac{\pi}{4}\right) & -1 & \exp \left(-j \frac{\pi}{4}\right) & j \\
1 & \exp \left(j \frac{\pi}{4}\right) & j & \exp \left(j \frac{3 \pi}{4}\right) \\
\exp \left(j \frac{3 \pi}{4}\right) & j & \exp \left(j \frac{\pi}{4}\right) & 1 \\
j & \exp \left(-j \frac{\pi}{4}\right) & -1 & \exp \left(j \frac{\pi}{4}\right)
\end{array}\right]
$$

For simplicity, we set the following terminology: In contrast to the ports at the antenna system, which are called the element ports, the ports at the input of the cascaded Butler network (at its left hand side in Fig. 1) are called the beam ports. Similar to the element ports, the beam ports are also numbered from left to right.

\section{Total Embedded Efficiencies}

Furthermore, to focus on the effects of beam-forming, we also need to abandon the influence of non-matched source impedances, i.e., we require $\mathbf{Z}_{s}=\mathbf{Z}_{\circ}$. In order to calculate the total embedded efficiency, one has to use a single non-zero entry excitation vector in (13) rendering the corresponding multiport matching efficiency. As a reminder, since the structure is assumed lossless, the multiport matching efficiencies equal the total embedded element efficiencies. To obtain these efficiencies at the element ports, it is sufficient to replace $\mathbf{S}_{12}$ and $\mathbf{S}_{21}$ in (2) by $\mathbf{I}$, and the associated $\mathbf{S}_{11}$ and $\mathbf{S}_{22}$ by $\mathbf{0}$, and consequently substitute the resultant into (13). After some simplifications, the desired parameters at the element ports become the diagonal entries in

$$
\mathbf{e}_{\text {tot }}=\operatorname{diag}\left(\mathbf{I}-\mathbf{S}_{a}{ }^{\dagger} \mathbf{S}_{a}\right) \quad \text { (element ports) }
$$

In a similar way, the total embedded efficiencies at the beam ports are achieved. Inserting the corresponding terms given in (14) into (13) and after some algebra, the required efficiencies at the beam ports become the diagonal entries in

$$
\mathbf{e}_{\text {tot }}=\operatorname{diag}\left(\mathbf{I}-\boldsymbol{\Gamma}_{1}^{\dagger} \boldsymbol{\Gamma}_{1}\right) \quad \text { (beam ports) }
$$

where

$$
\Gamma_{1}=\mathbf{T} \mathbf{S}_{a} \mathbf{T}^{\top}
$$

Using the above expression, the total embedded efficiencies of 4MP antenna versus frequency achieved from (15) and (16) are plotted in Fig. 4. These results belong to the case of $d=$ $0.2 \lambda_{\circ}$. Note that at this element separation, the neighboring elements present a fair amount of coupling, which is the source of their deviations from those in an isolated state. Although the efficiencies at the element and beam ports differ significantly, the arithmetic mean efficiencies in both cases are the same.

\section{Spatial Correlation}

Recall that we have restricted ourselves to lossless singlemode radiation structures. This restriction allows us to most conveniently calculate the spatial correlation in a uniform multipath environment at the element ports through the input network parameters [12]. To obtain the corresponding correlations at the beam ports, we use the expressions in [13, Equations $20 \& 31$ ]. One can show that the equations in (15) and (16) also yield similar results. Note that these correlations are credible in rich isotropic Rayleigh multipath environments

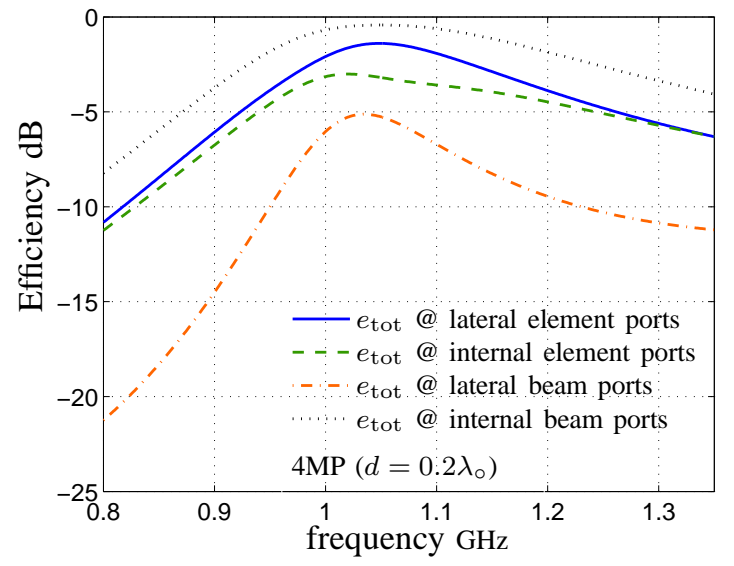

Fig. 4. Total embedded efficiencies versus frequency at element ports and beam ports for four collinear monopoles above a PEC plane.

wherein the term rich shall soon be clarified. In this paper, we study the square root of received powers' correlation and refer to it simply as correlation. It is known that the latter metric approximately equals the absolute value of complex correlation between the received voltage signals in a rich Rayleigh multipath environment [14], [15, Chapter 2].

As an example, Fig. 5 illustrates the correlations versus frequency for the 4MP antenna with $d=0.2 \lambda_{\circ}$. In this figure, the correlations at the element ports are denoted by $\rho$, whereas those at the beam ports are shown by $\varrho$. In addition to discrepancies in the total embedded efficiencies as seen before, the differences between the corresponding correlations at the element and beam ports are noticeable.

\section{E. Effective Diversity Gain}

In a multiport diversity antenna system used in a multipath environment, different performance metrics are derived from random received signals at its ports. In particular, the effective diversity gain is calculated through the cumulative distribution function (CDF) of the received random power signals [16]. Therefore, throughout this section, we are concerned about the simulation of received random power vectors. For this purpose, we use a classic approach in which a random multipath is emulated and then the embedded patterns of antennas are exposed to it. Consequently, the random received power signals are calculated, which are accordingly used to yield different metrics such as the effective diversity gain being henceforth referred to as diversity gain. The method has been used in [17] and later generalized and further detailed in [15, Chapter 4], [5]. Therefore, we avoid detailing it again and refer the interested readers to these references. Nevertheless, there are a few points that need to be cleared.

Concerning the angle of arrival (AoA) distribution, since a moving multiport antenna can have any arbitrary orientation in space, the effective distribution of AoA can on average be presumably uniform. The same kind of reasoning is applied equally to the effective polarization of the incoming waves. Thus, a uniform multipath environment of balanced polarization (i.e., isotropic multipath) stands as a generic 


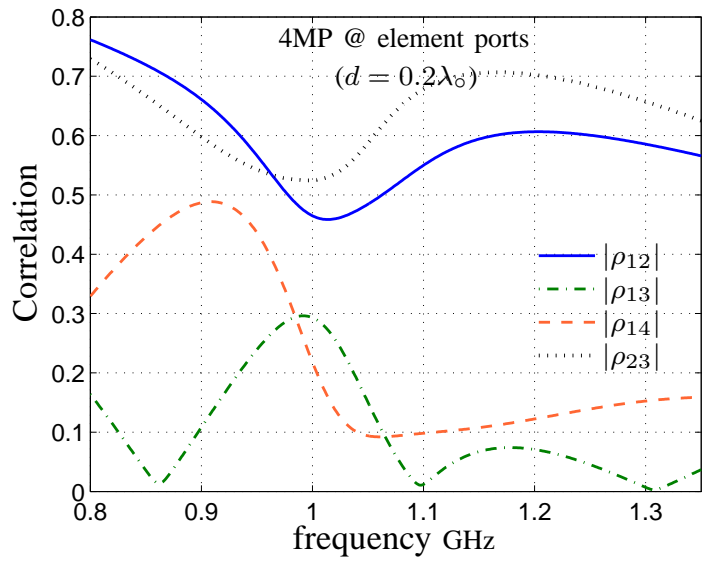

(a) Correlations at the element ports.

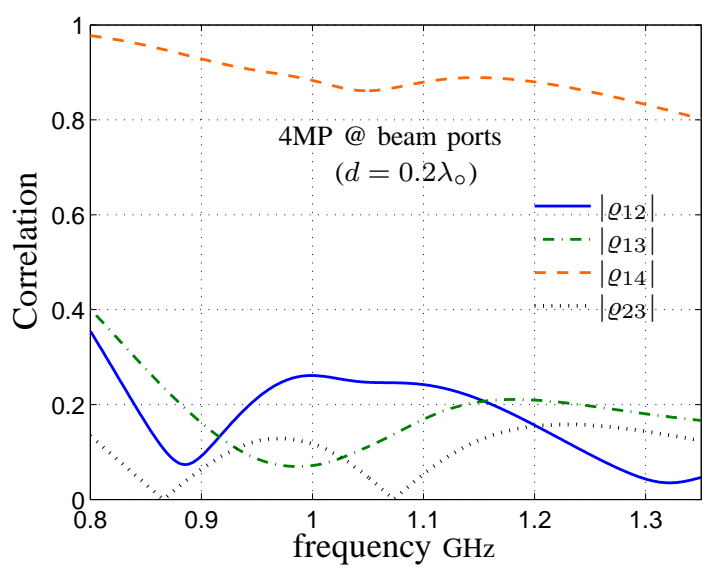

(b) Correlations at the beam ports.

Fig. 5. Correlations versus frequency for four monopoles above a PEC plane with $d=0.2 \lambda_{\circ}$.

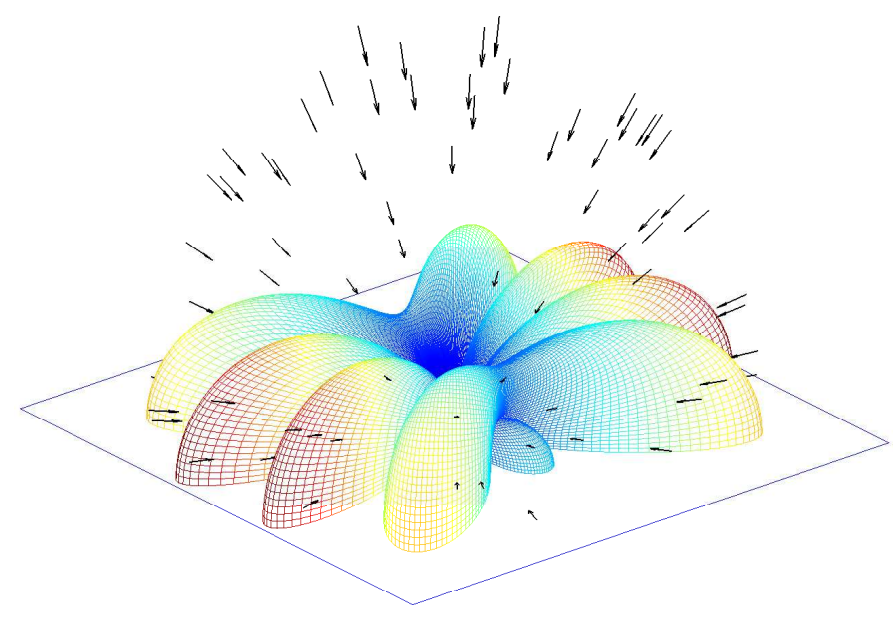

Fig. 6. A sample realization in which embedded far field functions of four monopoles (4MP) above a PEC $\left(d=0.5 \lambda_{\circ}\right)$ with a cascaded Butler network are exposed to 80 random incoming waves with uniform AoA within $\theta \in[0, \pi / 2]$ and $\psi \in[0,2 \pi]$.

representation of the actual multipath scenarios being retained for all simulations conducted in this paper. More importantly, the choice of uniform AoA will also nullify the impact of MEG variations caused by a cascaded Butler network. This serves our purpose best in the frame of the current study.

Furthermore, the number of random incoming waves in each realization indicates the richness of the multipath environment. This metric plays a central role in the remainder of this paper. In Fig. 6, a sample of such a scenario is depicted wherein 80 uncorrelated uniform random waves are incident upon the embedded patterns of 4MP antenna with a cascaded Butler network. In addition to this, the total number of realizations is associated with the accuracy of the results. Here, this number exceeds a million rendering a relative error of less than $0.05 \mathrm{~dB}$ accordingly to [18, Table 1].

As a final point, note that in parallel with the proposed radiation elements, we also need to expose an arbitrary reference antenna to the same set of incident waves at each scenario, and use its received average power for normalization purpose. In this study, we used an ideal isotropic antenna as the reference.

\section{Diversity Performance in Rich Multipath ENVIRONMENTS}

A rich multipath environment is an environment in which random received signals present a converged joint probability density function. In a rich isotropic Rayleigh environment, the diversity gain is a complex function of the correlation and the total embedded element efficiencies at different ports [18]. Figs. 4 and 5 illustrate the total embedded efficiencies and correlations versus frequency for 4MP antenna with a specific element separation. Apparently, at $f=1050 \mathrm{MHz}$ the 4MP structure presents an optimum mean radiation efficiency. Hence, we choose $f=1050 \mathrm{MHz}$ and study both radiation efficiencies and correlations versus element separation, $d$. The results are shown in Figs. 7 and 8, respectively. Once more, we observe significant differences between the associated metrics at the two sets of ports. Despite all these considerable discrepancies, since the rows and columns of $\mathbf{T}$ in (16) are orthogonal, the eigenvalues of the resultant covariance matrix is identical to those of (15). This requires the associated diversity gains be similar at the element and beam ports. In fact, our simulations verify this requirement. Thus, regardless of the amount of coupling among the radiation elements, in a rich isotropic environment, the ultimate diversity performances at the element and beam ports are the same. This is also known from measurements in [19]. Nevertheless, in non-rich multipath environment, the results are in general different which is the subject of the next section.

\section{Advantages of a Butler Network in NON-RICH MULTIPATH ENVIRONMENTS}

Up to now we have concluded that in a rich multipath environment the presence of a Butler network does not prove beneficial. However, the performance of the aforementioned network should also be examined in a non-rich scattering environment, where the number of random incident waves is finite.

To this end, and for a fair comparison between performances at the element and beam ports, we make some presumptions. 


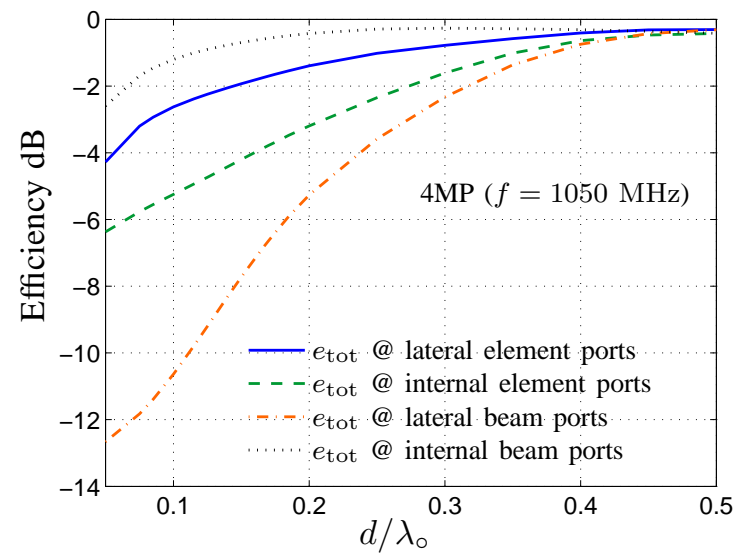

Fig. 7. Total embedded efficiencies versus element separations at element and beam ports for the 4MP antenna $(f=1050 \mathrm{MHz})$.

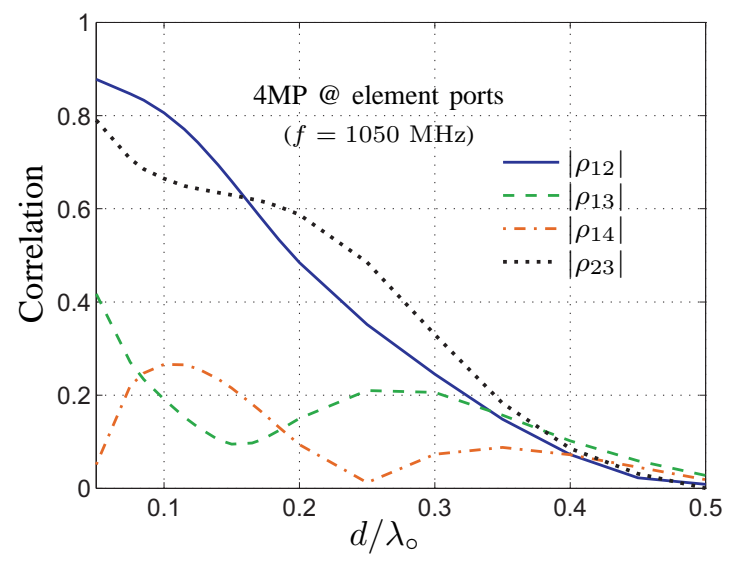

(a) Correlations at the element ports.

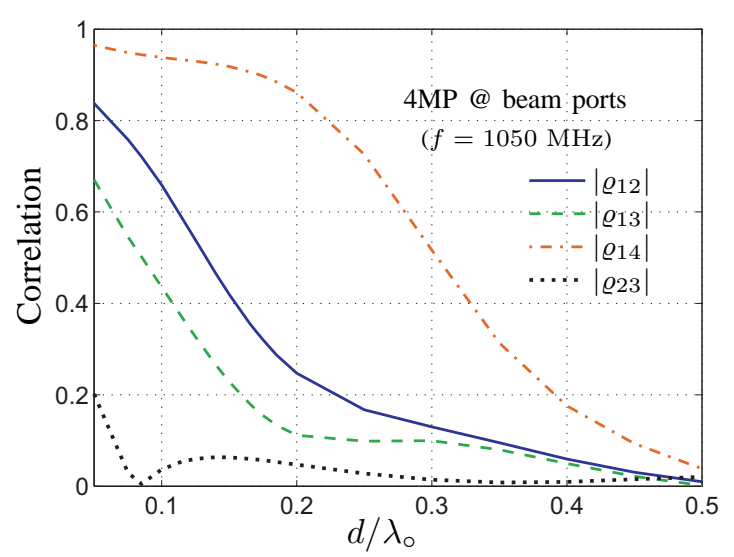

(b) Correlations at the beam ports.

Fig. 8. Spatial correlations for four monopoles above a PEC plane.
Recall that in a non-rich multipath environment, the joint distributions of random received signals do not converge. Convergence in joint distribution functions is linked to convergence in distributions of signals at different ports as well as convergence in their covariance matrix. To focus on the antenna characterization and alleviate its complexity, we assume that the distribution of the received signals at different ports is complex Gaussian. This assumption holds regardless of the number of incident waves in each realization. Thus, convergence in the joint distribution function will solely depend on convergence in the received signals' covariance matrix. The latter parameter depends on the shapes of the embedded patterns as well as the array configuration which are antenna engineers' concern. Bear in mind that the aforementioned constraint minimizes the least number of independent incoming waves yielding a converged joint distribution for the received signals. If we relax this restriction, a larger number of incident waves is necessary to cause convergence. This number is thus dependent not only on the antenna properties, but also on the distribution of the incoming waves which is beyond the intended scope.

To perform the simulation, we first choose a certain separation between the elements and a frequency of operation e.g., $d=0.5 \lambda_{\circ}$ and $f=1050 \mathrm{MHz}$. The $50 \Omega$ embedded patterns and the input parameters are achieved by the method of moment for both structures under study. It is known that proportional to the dimension of the Butler network, the directivity of the embedded patterns increases at the beam ports compared with those of the element ports [1]. Here, the variable is the number of incident waves in each realization. As an interesting start, let us study the CDFs of the simulated received signals by the 4MP antenna $\left(d=0.2 \lambda_{\circ}\right)$ for two different numbers of incident waves, e.g., $K=5$ and $K=30$. Fig 9 illustrates the results. Since the number of incident waves does not affect the normalized average received power, under the assumption of Rayleigh distributed received signals, the CDFs of the signals at different ports are independent of this number. Furthermore, due to the symmetry of the structure, the two lateral and internal ports have similar CDF curves. Nevertheless, the CDF curve of the maximum ratio combining (MRC) diversity signal depends on the number of incident waves. While with $K=30$ number of incident waves, the CDFs of the diversity signals at both element and beam ports are almost the same, for $K=5$ the CDF of the diversity signal associated with the beam ports shows better performance compared to that of the element ports. Fig. 10, shows the MRC diversity gain at the $1 \% \mathrm{CDF}$ level for this particular case of $d=0.2 \lambda_{\circ}$ versus the number of incident waves in each realization. Similar results associated with 4HD antennas $\left(d=0.5 \lambda_{\circ}\right)$ are shown in Fig. 11 .

It is important to stress that the corresponding total embedded efficiencies simulated in this study are independent of the number of independent incident waves. But, the covariance matrix of the received signals varies with respect to this variable. Fig. 12 shows convergence in correlations at element and beam ports for 4HD antennas at $d=0.5 \lambda_{\circ}$ and $f=1050$ MHz. An identical observation can be made for correlations in 4MP antennas which is neglected here for the sake of conciseness.

From a diversity performance point of view, in these sim- 
TABLE I

RICHNESS THRESHOLD VERSUS ELEMENT SEPARATION AT $-0.5 \mathrm{~dB}$ LEVEL FOR 4HD ANTENNAS $(f=1050 \mathrm{MHZ})$.

\begin{tabular}{ccc}
\hline & \multicolumn{2}{c}{ Richness Threshold (waves) } \\
\cline { 2 - 3 } Element separation & Element ports & Beam ports \\
\hline $0.05 \lambda_{\circ}$ & 15 & 13 \\
$0.10 \lambda_{\circ}$ & 19 & 6 \\
$0.15 \lambda_{\circ}$ & 26 & 7 \\
$0.20 \lambda_{\circ}$ & 25 & 10 \\
$0.25 \lambda_{\circ}$ & 27 & 13 \\
$0.30 \lambda_{\circ}$ & 32 & 13 \\
$0.40 \lambda_{\circ}$ & 31 & 7 \\
\hline
\end{tabular}

ulations the optimum diversity gain cannot be realized unless the covariance matrix of the received signals converges. This occurs when the number of incident waves exceeds a threshold. As the number of the incident wave increases beyond this threshold, no extra diversity gain can be achieved. In our preliminary work, we referred to this novel performance metric as the richness threshold [5]. If the distribution of the incoming waves is complex Gaussian, richness threshold becomes a unique measure rendering some information about the overall performance of a multiport diversity antenna system. As a criterion, the less the richness threshold, the better the diversity system performance.

In order to quantify the enhancement caused by the Butler network, we study the richness threshold. For this purpose let us choose the threshold as the number of independent incident waves by which the diversity gain exceeds $-0.5 \mathrm{~dB}$ level from its asymptotic (or converged) value. Fig. 13 illustrates this metric versus element separation for 4MP antenna at $f=1050$ MHz. There is no doubt that the Butler network reduces the richness threshold considerably. Based on this figure, the best performance belongs to the cases of $d=0.4 \lambda_{\circ}-0.5 \lambda_{\circ}$ where only four independent incident waves are sufficient for the antenna to realize $90 \%$ of its optimum performance. For the sake of comparison, similar results for 4HD antenna are summarized in Table I. Regardless of the element separation and in the absence of a Butler network, apparently the 4HD antenna requires more number of independent waves to represent an effective diversity antenna.

In general, the significance of richness threshold may be understood more when one recalls that in a typical multipath environment, the frequently reported number of incoming waves at an outdoor receiving antenna is five or six [20], [21], [22]. A number of 20 incoming waves in an indoor scattering environment seems also presumably acceptable [23]. But, for numerous available multiport antennas, this number of incident waves is insufficient to realize the best diversity performance. Therefore, in practice, most of the multiport antennas are working below their richness threshold and thus cannot realize their optimum performance.

\section{CONCLUSION}

This paper provides a general - yet compact - expression for calculation of multiport matching efficiencies in the presence of a microwave network. In a lossless structure, the multiport matching efficiencies equal the corresponding total embedded

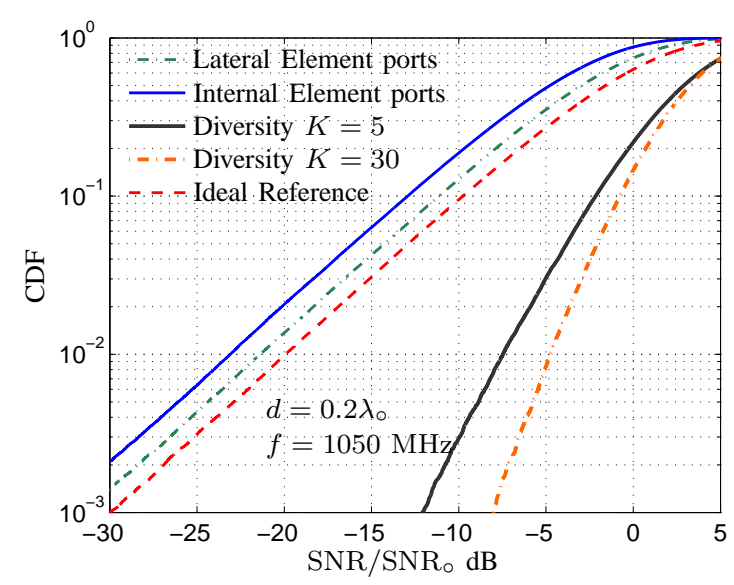

(a) $\mathrm{CDF}$ curves at the element ports for $4 \mathrm{MP}$ antenna.

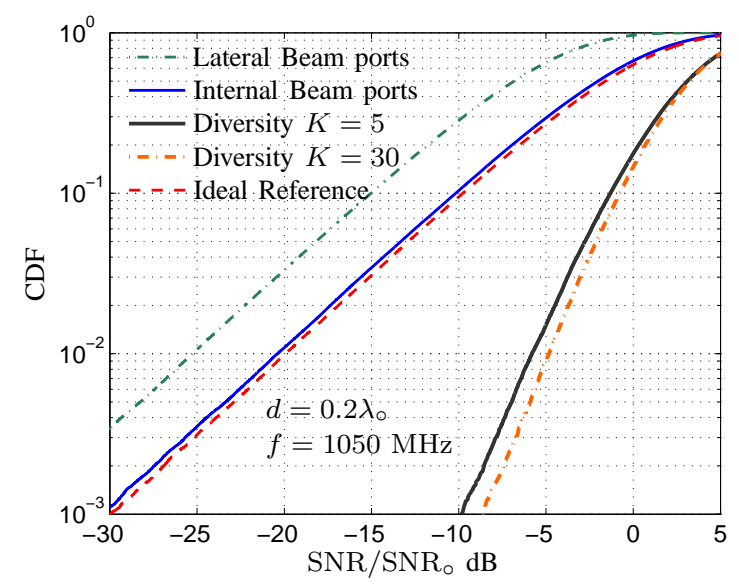

(b) CDF curves at the beam ports for 4MP antenna.

Fig. 9. CDFs of the received signals and diversity signals for four monopoles above a PEC plane. $K$ is the number of incident waves and $\mathrm{SNR}_{\circ}$ is the average received power by the reference antenna.

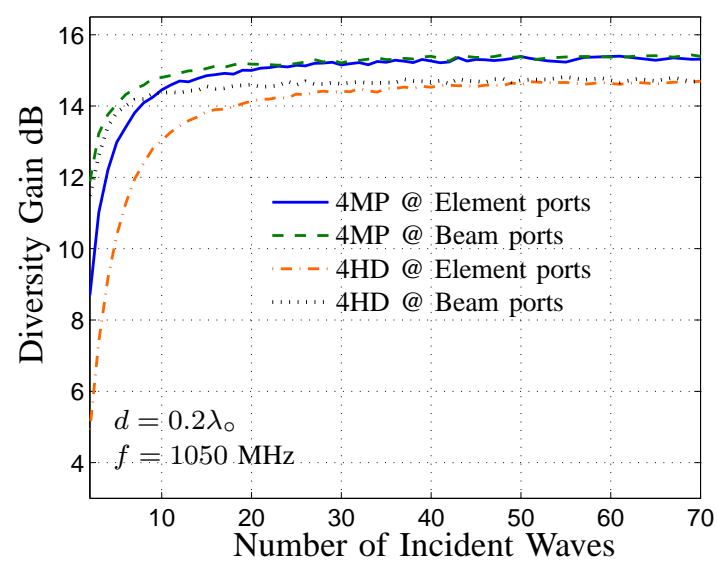

Fig. 10. MRC Effective diversity gain at the $1 \% \mathrm{CDF}$ level versus the number of incident waves for the two antennas under test with $d=0.2 \lambda_{\circ}$.

efficiencies. These efficiencies are used for normalization of the embedded pattern in the presence of an arbitrary cascaded network. The main purpose of this paper is to investigate the impacts of a cascaded Butler network in a diversity antenna 


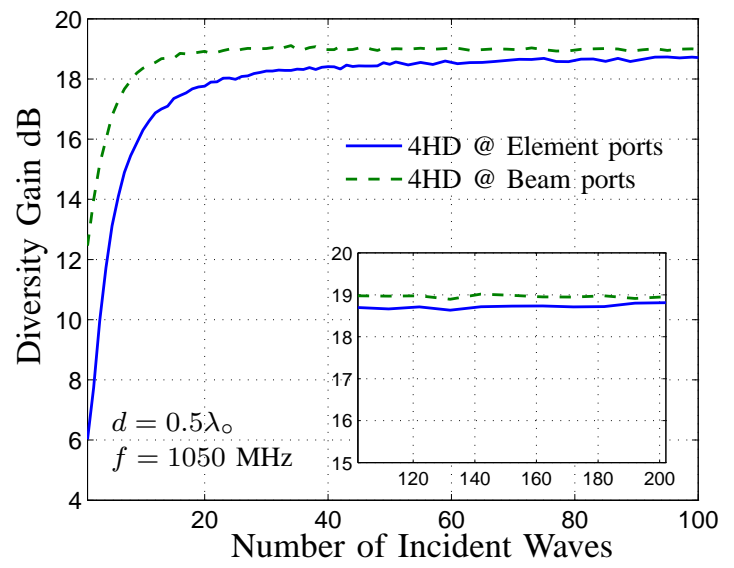

Fig. 11. MRC Effective diversity gain at the $1 \% \mathrm{CDF}$ level versus the number of incident waves for the 4HD antenna under test with $d=0.5 \lambda_{\circ}$. The labels and the legends of the internal figure are the same as those in the main figure.

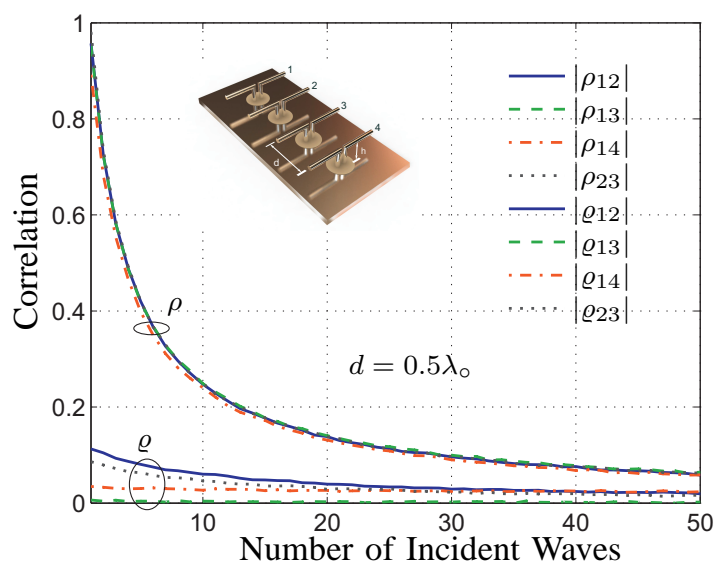

Fig. 12. Convergence in correlation for 4HD antenna with $d=0.5 \lambda_{\circ} . \rho$ signifies correlations at the element ports, whereas $\varrho$ those of the beam ports.

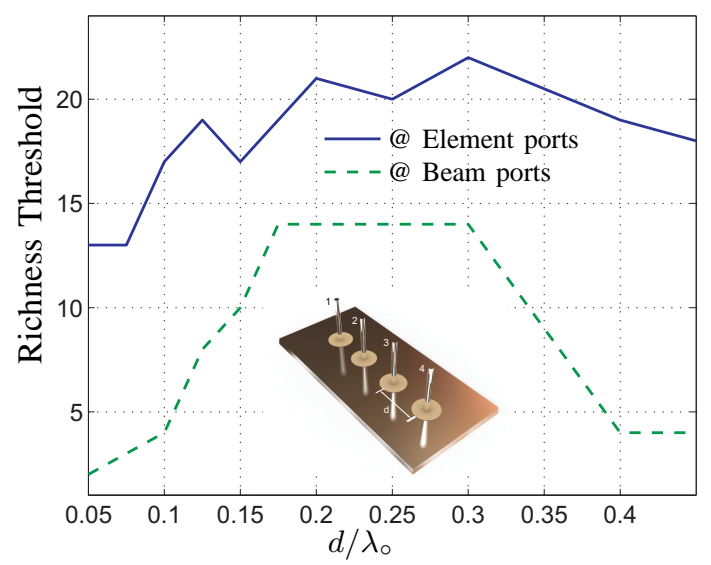

Fig. 13. Richness threshold for 4MP antenna at $-0.5 \mathrm{~dB}$ level versus element separation at the element port and beam ports $(f=1050 \mathrm{MHz})$.

system. Limiting ourselves to lossless structures in a uniform multipath environment, for four monopoles above a PEC plane, we present a study of correlation and total embedded element efficiencies both in the presence and absence of a cascaded Butler network. The results show considerable differences between the aforementioned metrics. Nevertheless, in a rich isotropic multipath, regardless of the frequency of operation and the amount of coupling between different elements, we have an identical diversity performance at both element and beam ports. In contrast, in a non-rich multipath environment, which represents actual scenarios better, the two proposed multiport antennas show a reduced performance. The deteriorated performance in the non-rich multipath is linked with the convergence in covariance matrix of the received signals at different ports. We show by numerical simulation that under this circumstance the cascaded Butler network to the antenna system proves beneficial. To quantify the advantage of the cascaded Butler network, we study the richness threshold at the element and beam ports, which is a novel performance metric. In general, the lower the richness threshold, the better the antenna's overall diversity performance. In this regard, the cascaded Butler network decreases the richness threshold and is thus quite advantageous in most practical environments wherein the typical number of incident waves is less than twenty. We emphasize that the results presented in this paper is under presumption of received signals of Rayleigh distribution, regardless of the number of incident waves. A further study is required to reveal the impact of a cascaded Butler network upon convergence in distribution of the random received signals.

\section{ACKNOWLEDGMENT}

The computations were performed on C3SE computing resources in Gothenburg, Sweden.

\section{REFERENCES}

[1] A. Molisch and X. Zhang, "FFT-based hybrid antenna selection schemes for spatially correlated MIMO channels," IEEE Communications Letters, vol. 8, no. 1, pp. 36-38, January 2004.

[2] A. Grau, J. Romeu, and F. De Flaviis, "On the diversity gain using a Butler matrix in fading MIMO environments," in IEEE/ACES International Conference on Wireless Communications and Applied Computational Electromagnetics, April 2005.

[3] A. Grau, J. Romeu, L. Jofre, and F. De Flaviis, "On the MIMO capacity using a Butler matrix with circular arrays in fading indoor environments," in IEEE Antennas and Propagation Society International Symposium, vol. 2A, July 2005.

[4] A. Grau, J. Romeu, S. Blanch, L. Jofre, and F. De Flaviis, "Optimization of linear multielement antennas for selection combining by means of a butler matrix in different MIMO environments," IEEE Transactions on Antennas and Propagation, vol. 54, no. 11, pp. 3251-3264, November 2006.

[5] N. Jamaly, M. Iftikhar, and Y. Rahmat-Samii, "Performance evaluation of diversity antennas in multipath environments of finite richness," in 2012 Proceedings of the 6th European Conference on Antennas and Propagation (EUCAP), March 2012.

[6] D. M. Pozar, Microwave Engineering, 3rd ed. John Wiley \& Sons, 2005.

[7] N. Jamaly and A. Derneryd, "Efficiency characterisation of multi-port antennas," Electronics Letters, vol. 48, no. 4, pp. 196-198, February 2012.

[8] - , Multi-port matching efficiency in antenna systems with cascaded networks," in 15th International Symposium on Antenna Technology and Applied Electromagnetics (ANTEM), June 2012.

[9] W. Wiesbeck and E. Heidrich, "Wide-band multiport antenna characterization by polarimetric RCS measurements," IEEE Transactions on Antennas and Propagation, vol. 46, no. 3, pp. 341-350, March 1998. 
[10] M. Manteghi and Y. Rahmat-Samii, "Multiport characteristics of a wide-band cavity backed annular patch antenna for multipolarization operations," IEEE Transactions on Antennas and Propagation, vol. 53, no. 1, pp. 466-474, January 2005.

[11] G. P. Riblet, "Simplified 8-port S-matrix of the general reciprocal lossless matched 4-way divider with output port isolation," IEEE Microwave and Guided Wave Letters, vol. 5, no. 11, pp. 365-367, November 1995.

[12] S. Blanch, J. Romeu, and I. Corbella, "Exact representation of antenna system diversity performance from input parameter description," Electronics Letters, vol. 39, no. 9, pp. 705-707, May 2003.

[13] J. W. Wallace and M. A. Jensen, "Termination-dependent diversity performance of coupled antennas: Network theory analysis," IEEE Transactions on Antennas and Propagation, vol. 52, no. 1, pp. 98-105, January 2004.

[14] R. Vaughan and J. Andersen, "Antenna diversity in mobile communications," IEEE Transactions on Vehicular Technology, vol. 36, no. 4, pp. 149-172, November 1987

[15] N. Jamaly, "Spatial Characterization of Multi-element Antennas," Chalmers University of Technology, Sweden, Tech. Rep. R002/2011, February 2011. [Online]. Available: http://publications.lib.chalmers.se/records/fulltext/137635.pdf

[16] P.-S. Kildal, K. Rosengren, J. Byun, and J. Lee, "Definition of effective diversity gain and how to measure it in a reverberation chamber," Microwave and Optical Technology Letters, vol. 34, no. 1, pp. 56-59, July 2002.

[17] K. Rosengren and P.-S. Kildal, "Radiation efficiency, correlation, diversity gain and capacity of six monopole antenna array for a MIMO system: Theory, simulation and measurement in reverberation chamber," Proceedings IEE, Microwaves Antennas and Propagation, vol. 152, no. 1, pp. 7-16, 2005, see also Erratum published in August 2006.

[18] N. Jamaly, P.-S. Kildal, and J. Carlsson, "Compact formulas for diversity gain of two-port antennas," IEEE Antennas and Wireless Propagation Letters, vol. 9, pp. $970-973,2010$.

[19] N. Jamaly, C. Gomez-Calero, P.-S. Kildal, J. Carlsson, and A. Wolfgang, "Study of excitation on beam ports versus element ports in performance evaluation of diversity and MIMO arrays," in 2009 Proceedings of the Third European Conference on Antennas and Propagation (EuCAP), March 2009.

[20] F. Ikegami and S. Yoshida, "Analysis of multipath propagation structure in urban mobile radio environments," IEEE Transactions on Antennas and Propagation, vol. 28, no. 4, pp. 531-537, 1977.

[21] S. Sakagami, "Experimental results on multipath propagation in 900 $\mathrm{MHz}$ mobile communication path-amplitude-frequency characteristics and angle of arrival," IEICE Japan Transactions, vol. J70-B, no. 12, pp. $1522-1528,1987$.

[22] T. Taga, "Analysis for mean effective gain of mobile antennas in land mobile radio environments," IEEE Transactions on Vehicular Technology, vol. 39, no. 2, pp. 117-131, 1990.

[23] G. Calcev, D. Chizhik, B. Goransson, S. Howard, H. Huang, A. Kogiantis, A. Molisch, A. Moustakas, D. Reed, and H. Xu, "A wideband spatial channel model for system-wide simulations," IEEE Transactions on Vehicular Technology, vol. 56, no. 2, pp. 389-403, March 2007.

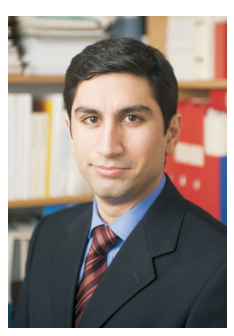

Nima Jamaly is originally from northern Iran. He started his BSc studies in Iran University of Science and Technology in Tehran, and eventually received a $\mathrm{PhD}$ degree in multiport antenna systems from Chalmers University of Technology, Sweden. In total, he holds five degrees from different universities in several areas of electrical engineering in particular computational electromagnetics, microwave and RF circuit design, fiber optic communication systems, array antennas, signal processing, and reverberation chambers. In 2006 he was with the Laboratory of Electromagnetics and Acoustics, Swiss Federal Institute of Technology, Lausanne, Switzerland. In 2007, he joined the Microwave and Photonics Laboratory in Royal Institute of Technology, Stockholm, Sweden. In 20082009, he was with the Antenna Group, Chalmers University of Technology. In 2010, he pursued his research as a visiting PhD student in Antenna Research, Analysis and Measurement Laboratory, University of California Los Angeles. During the last few years, Nima has been involved in several European projects in modern wireless communication systems. Since early 2013, he is a researcher in Communication Systems Group at Chalmers. His current research associates with design and optimization of predictor antenna systems in moving relays for new generation of mobile communication systems.

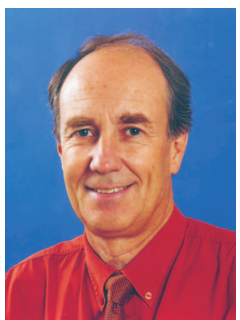

Anders Derneryd received the $\mathrm{MSc}$ and $\mathrm{PhD}$ degrees in electrical engineering from Chalmers University of Technology, Gothenburg, Sweden, in 1971 and 1976, respectively. $\mathrm{He}$ is an Expert in Antenna Technology at Antenna System Technology, Ericsson Research, Gothenburg, Sweden working on adaptive and active array antenna systems for future wireless communication applications. His research interests include array antennas for base stations, multi-beam antennas for point-to-multipoint radio links, and multiple-antennas for terminal applications. Since 1999, he is also an Adjunct Professor in Antenna Technology at Lund Institute of Technology, Lund, Sweden.

From 1978 until 1992 Dr. Derneryd was a Member of the Antenna Department at Ericsson Radar Electronics, Mölndal, Sweden working on space and military projects. At the formation of Saab Ericsson Space in 1992, he joined its Antenna Department and in 1995 he moved to Ericsson Microwave Systems and the departments of GSM \& Antenna Products and later to the Core Unit Antenna Technology. He was responsible for studies and electrical design of a number of slotted waveguide array antennas, microstrip array antennas, and dichroic subreflectors. He has also been working with engineering management and electrical design of array antennas for remote sensing and communication satellites under contracts from the European Space Agency.

Dr. Derneryd pursued his interest in microstrip antennas at the Rome Air Development Center, Hancom AFB, MA, in a Post-Doctoral Program during 1976 and as a Visiting Research Associate at the ECE Department, University of Colorado, from 1986 to 1987. In 1992 he spent some months at Ericsson Inc in RTP, NC, USA, performing studies on array antenna systems for mobile communication satellites.

Dr. Derneryd has published and presented more than 100 research and application papers within the antenna field. He has also contributed to a number of book chapters on antenna applications. He is the co-inventor of approximately 30 patents or patents pending within the field of antenna systems for mobile communications.

Dr. Derneryd is a Member of Section B of the Swedish National Committee of URSI. He has been a Swedish expert at a number of European COST actions. 


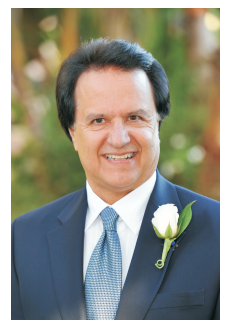

Yahya Rahmat-Samii (S'73-M'75-SM'79-F'85) is a Distinguished Professor, holder of the Northrop Grumman Chair in Electromagnetics, member of the US National Academy of Engineering (NAE) and past Chairman of the Electrical Engineering Department, University of California, Los Angeles (UCLA). He was a Senior Research Scientist with the National Aeronautics and Space Administration (NASA) Jet Propulsion Laboratory (JPL), California Institute of Technology prior to joining UCLA in 1989. In summer 1986, he was a Guest Professor with the Technical University of Denmark (TUD). He has also been a consultant to numerous aerospace and wireless companies. He has been Editor and Guest editor of numerous technical journals and books. He has authored and coauthored over 800 technical journal and conference papers and has written 30 book chapters. He is a coauthor of Electromagnetic Band Gap Structures in Antenna Engineering (New York: Cambridge, 2009), Implanted Antennas in Medical Wireless Communications (Morgan \& Claypool Publishers, 2006), Electromagnetic Optimization by Genetic Algorithms (New York: Wiley, 1999), and Impedance Boundary Conditions in Electromagnetics (New York: Taylor \& Francis, 1995). He has received several patents. He has had pioneering research contributions in diverse areas of electromagnetics, antennas, measurement and diagnostics techniques, numerical and asymptotic methods, satellite and personal communications, human/antenna interactions, RFID and implanted antennas in medical applications, frequency selective surfaces, electromagnetic band-gap structures, applications of the genetic algorithms and particle swarm optimization, etc., (visit http://www. antlab.ee.ucla.edu/).

Dr. Rahmat-Samii is a Fellow of the Institute of Advances in Engineering (IAE), a Fellow of AMTA and a Fellow ACES. He is a member of Commissions $\mathrm{A}, \mathrm{B}, \mathrm{J}$ and $\mathrm{K}$ of USNC-URSI, the Antenna Measurement Techniques Association (AMTA), Sigma Xi, Eta Kappa Nu and the Electromagnetics Academy. He was Vice-President and President of the IEEE Antennas and Propagation Society in 1994 and 1995, respectively. He was appointed an IEEE AP-S Distinguished Lecturer and presented lectures internationally. He was a member of the Strategic Planning and Review Committee (SPARC) of the IEEE. He was the IEEE AP-S Los Angeles Chapter Chairman (19871989); his chapter won the best chapter awards in two consecutive years. He is listed in Who's Who in America, Who's Who in Frontiers of Science and Technology and Who's Who in Engineering. He has been the plenary and millennium session speaker at numerous national and international symposia. $\mathrm{He}$ has been the organizer and presenter of many successful short courses worldwide. He was a Directors and Vice President of AMTA for three years. $\mathrm{He}$ has been Chairman and Co-chairman of several national and international symposia. He was a member of the University of California at Los Angeles (UCLA) Graduate council for three years. He was the chair of USNC-URSI for the period of 2009-2011.

For his contributions, Dr. Rahmat-Samii has received numerous NASA and JPL Certificates of Recognition. In 1984, he received the Henry Booker Award from URSI, which is given triennially to the most outstanding young radio scientist in North America. Since 1987, he has been designated every three years as one of the Academy of Science's Research Council Representatives to the URSI General Assemblies held in various parts of the world. $\mathrm{He}$ was also invited speaker to address the URSI 75th anniversary in Belgium. In 1992 and 1995, he received the Best Application Paper Prize Award (Wheeler Award) for papers published in 1991 and 1993 IEEE Transactions on Antennas and Propagation. In 1999, he received the University of Illinois ECE Distinguished Alumni Award. In 2000, Prof. Rahmat-Samii received the IEEE Third Millennium Medal and the AMTA Distinguished Achievement Award. In 2001, Rahmat-Samii received an Honorary Doctorate in applied physics from the University of Santiago de Compostela, Spain. In 2001, he became a Foreign Member of the Royal Flemish Academy of Belgium for Science and the Arts. In 2002, he received the Technical Excellence Award from JPL. He received the 2005 URSI Booker Gold Medal presented at the URSI General Assembly. He is the recipient of the 2007 Chen-To Tai Distinguished Educator Award of the IEEE Antennas and Propagation Society. In 2008, he was elected to the membership of the US National Academy of Engineering (NAE). In 2009, he was selected to receive the IEEE Antennas and Propagation Society highest award, Distinguished Achievement Award, for his outstanding career contributions. He is the recipient of the 2010 UCLA School of Engineering Lockheed Martin Excellence in Teaching Award, the 2011 UCLA Distinguished Teaching Award and the 2011 IEEE Electromagnetics Award. Prof. Rahmat-Samii is the designer of the IEEE AP$\mathrm{S}$ logo which is displayed on all IEEE AP-S publications. 